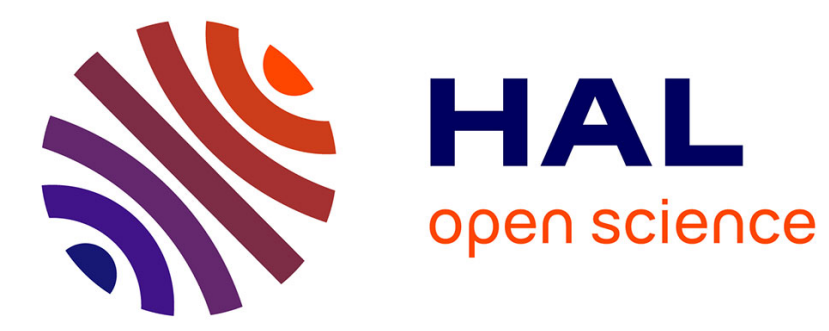

\title{
Psychopathologie et réinsertion professionnelle
}

Bernard Pachoud, Marli Nascimento Stieffatre

\section{To cite this version:}

Bernard Pachoud, Marli Nascimento Stieffatre. Psychopathologie et réinsertion professionnelle: Une clinique pluridimensionnelle requérant des ressources théoriques pluridisciplinaires. Cliniques méditerranéennes, 2005, Précarité, exclusion, abandon, 2 (72), pp.147-171. 10.3917/cm.072.0147 . hal01505636

\section{HAL Id: hal-01505636 \\ https://hal.science/hal-01505636}

Submitted on 11 Apr 2017

HAL is a multi-disciplinary open access archive for the deposit and dissemination of scientific research documents, whether they are published or not. The documents may come from teaching and research institutions in France or abroad, or from public or private research centers.
L'archive ouverte pluridisciplinaire HAL, est destinée au dépôt et à la diffusion de documents scientifiques de niveau recherche, publiés ou non, émanant des établissements d'enseignement et de recherche français ou étrangers, des laboratoires publics ou privés. 


\section{PSYCHOPATHOLOGIE ET RÉINSERTION PROFESSIONNELLE}

Une clinique pluridimensionnelle requérant des ressources théoriques

pluridisciplinaires

Bernard Pachoud, Marli Nascimento Stieffatre

ERES | « Cliniques méditerranéennes »

2005/2 $\mathrm{n}^{0} 72 \mid$ pages 147 à 171

ISSN 0762-7491

ISBN 2749204054

Article disponible en ligne à l'adresse :

http://www.cairn.info/revue-cliniques-mediterraneennes-2005-2-page-147.htm

\section{Pour citer cet article :}

Bernard Pachoud, Marli Nascimento Stieffatre, «Psychopathologie et réinsertion professionnelle. Une clinique pluridimensionnelle requérant des ressources théoriques pluridisciplinaires », Cliniques méditerranéennes 2005/2 ( $\left.\mathrm{n}^{\circ} 72\right)$, p. 147-171.

DOI 10.3917/cm.072.0147

Distribution électronique Cairn.info pour ERES.

(C) ERES. Tous droits réservés pour tous pays.

La reproduction ou représentation de cet article, notamment par photocopie, n'est autorisée que dans les limites des conditions générales d'utilisation du site ou, le cas échéant, des conditions générales de la licence souscrite par votre établissement. Toute autre reproduction ou représentation, en tout ou partie, sous quelque forme et de quelque manière que ce soit, est interdite sauf accord préalable et écrit de l'éditeur, en dehors des cas prévus par la législation en vigueur en France. Il est précisé que son stockage dans une base de données est également interdit. 


\title{
Bernard Pachoud et Marli Nascimento Stieffatre
}

\author{
Psychopathologie \\ et réinsertion professionnelle \\ Une clinique pluridimensionnelle
}

requérant des ressources théoriques pluridisciplinaires

\section{DIVERSITÉ DES PRATIQUES DE SOUTIEN}

ET D'ACCOMPAGNEMENT À L'INSERTION PROFESSIONNELLE

L'insertion professionnelle des malades mentaux reste une question d'actualité, qui renvoie à une diversité de pratiques actuellement en plein développement, visant à promouvoir, soutenir et accompagner le retour à l'emploi de personnes ayant présenté des troubles psychiatriques et, le plus souvent, continuant de bénéficier de soins. Ce souci de réinsertion professionnelle des patients psychiatriques s'inscrit dans le cadre plus général des efforts visant à la réinsertion sociale de ces personnes, le travail apparaissant tantôt comme un aspect privilégié mais pas nécessairement requis de la réinsertion, tantôt au contraire comme une des conditions à une insertion de qualité. C'est donc sur ces pratiques de préparation et de soutien à la réinsertion qu'il apparaît souhaitable de s'interroger, sur leurs moyens et leurs méthodes, mais aussi sur ceux qui en sont les acteurs, sur les contextes (institutionnels, administratifs, législatifs...) qui en orientent le développement, et enfin sur les discours et constructions théoriques qui accompagnent, légitiment et influencent ces pratiques. C'est aussi en fonction de leurs « bénéficiaires » que ces pratiques nous intéressent, ce qui suscite d'autres questions : 
quels sont, pour eux, les effets de ces pratiques ? Et en retour, comment s'insèrent-ils dans ces pratiques, quelles places, quels rôles y occupent-ils ? Quels sens prennent-elles pour eux ? Quelles dynamiques suscitent-elles ?

Avant d'en venir aux détails, il convient de remarquer d'emblée que la diversité de ces pratiques d'aide à la réinsertion tient à la variété des besoins selon les patients et à la progressivité graduelle de leur réinsertion. Entre l'hôpital et l'entreprise, pour les patients qui ne sont pas ou plus insérés professionnellement, le chemin exige le plus souvent des étapes. C'est par conséquent ce parcours d'insertion qu'il faudrait pouvoir considérer dans son déploiement, en étant attentif aux étapes proposées, en s'interrogeant sur celles qui pourraient faire défaut, et en s'intéressant pour chacune de ces étapes à leurs apports spécifiques, aux effets engendrés... À défaut de pouvoir couvrir ici un si large champ de recherches, qui pourtant serait le plus à même de rendre compte de la variété des pratiques soutenant la réinsertion, nous limiterons nos réflexions et remarques à l'une des étapes du parcours d'insertion, celle du bilan d'aptitude et d'orientation, prestation sollicitée par les structures chargées spécifiquement de la réinsertion (COTOREP, Capemploi...), et à laquelle nous avons eu l'occasion de participer depuis environ trois ans.

Nous privilégierons ici un questionnement théorique plutôt qu'une véritable analyse de pratique ou l'examen de cas cliniques, et commencerons par nous interroger sur le ou plutôt les arrière-plans théoriques susceptibles de contribuer à l'analyse (et par suite au développement) de ces pratiques de soutien à la réinsertion des " handicapés psychiques ». Disons-le d'emblée, nous plaiderons pour que cette pratique pluridimensionnelle puisse s'appuyer sur une théorisation pluridisciplinaire. S'interroger sur l'insertion professionnelle des handicapés psychiques, $c^{\prime}$ est chercher à comprendre à la fois ce qui fait difficulté ou obstacle à une telle insertion, mais aussi ce que peut leur apporter, au plan psychologique ou personnel, une telle insertion professionnelle. Or ces difficultés aussi bien que ces ressources liées spécifiquement au travail peuvent être éclairées par l'analyse de la spécificité du travail parmi les activités humaines, de sa " fonction psychologique » pour reprendre à Y. Clot cette désignation d'une direction de recherche déjà richement documentée (Clot, 1999). Autrement dit, pour préciser les difficultés spécifiques mais aussi l'intérêt, pour le handicapé psychique, de l'insertion par le travail, il nous apparaît pertinent de rechercher du côté du travail (d'une théorie des propriétés et ressources propres à ce type d'activité) et pas seulement du côté du sujet (de ses fragilités, de ses dysfonctionnements psychiques), comme cela tend à être le réflexe en psychopathologie. Autrement dit encore, il s'agit de dégager des analyses de ce qui se joue dans le travail, 
ce qui peut contribuer à mieux problématiser ce que représente le travail pour une personne ayant présenté des troubles mentaux.

\section{LE CONTEXTE DE DÉVELOPPEMENT}

DES PRATIQUES DE SOUTIEN À L'INSERTION PROFESSIONNELLE

Adopter un point de départ factuel, en l'occurrence le constat de développement tendanciel des pratiques d'aide à l'insertion professionnelle des personnes ayant présenté des troubles psychiques, conduit à s'interroger sur la genèse de cette situation. En France, cette situation résulte notamment de choix opérés en matière de politique sanitaire et sociale, choix politiques concrétisés par des dispositions législatives fixant le cadre et les ressources allouées pour le soutien à l'insertion sociale et professionnelle des personnes handicapées.

La loi d'orientation en faveur des personnes handicapées, du 30 juin 1975, dans son volet pour les adultes, fixe un ensemble de dispositions liées à trois axes prioritaires : l'emploi, les ressources, l'hébergement. La loi proclame le droit au travail pour les personnes handicapées et prend des dispositions pour l'insertion au travail de ceux qui ne peuvent travailler en milieu ordinaire, ce qui sera précisé les années suivantes par la promulgation des statuts des centres d'aide par le travail (décret de 1977) et des ateliers protégés (arrêté de 1978). En matière de ressources, il est assuré aux personnes handicapées exerçant une activité professionnelle (en milieu protégé en particulier) une garantie de ressources, et pour celles qui ne peuvent travailler sont prévues des allocations. Pour bénéficier de ces prestations, il importe d'être reconnu handicapé par la commission technique d'orientation et de reclassement professionnel (COTOREP), instituée dans chaque département par cette même loi de 1975. Cette commission comporte deux sections, la première reconnaît la qualité de travailleur handicapé, évalue la capacité professionnelle, se prononce sur le reclassement et oriente vers une filière professionnelle ou de formation appropriée ; la deuxième section évalue le taux d'incapacité, décide de l'attribution de la carte d'invalidité et des allocations.

En matière d'insertion professionnelle, la politique de l'emploi concernant les handicapés est spécifiée par la loi du 10 juillet 1987, qui instaure une obligation d'emploi des travailleurs handicapés et assimilés pour tous les établissements de vingt salariés au moins, dans la proportion de $6 \%$ de l'effectif total de leurs salariés. Les entreprises qui ne satisfont pas cette exigence doivent en compensation verser une contribution, calculée sur l'effectif de l'entreprise, à l'AGEFIPH (Association de gestion du fonds pour l'insertion professionnelle des personnes handicapées). Cette instance de gestion des fonds pour l'insertion est contrôlée paritairement par des représentants des entre- 
prises, des salariés, des handicapés et de l'État. Elle soutient, favorise le développement et finance, partiellement ou totalement, nombre de dispositifs de soutien à la réinsertion.

Ce cadre législatif concernant l'insertion sociale et professionnelle a donc pour effet d'assujettir les prestations d'aide à l'insertion professionnelle à l'obtention du statut de "handicapé ", ce qui dépend des commissions départementales (СОтОREP). Quant aux différentes prestations, elles sont dispensées ou assurées non directement par les structures de soins mais essentiellement par des structures relevant du secteur social ou médico-social. Ce contexte administratif et légal a donc largement contribué à ce qu'on peut appeler le transfert de la prise en charge des personnes en quête de réinsertion, du secteur sanitaire au secteur social ou médico-social.

Cette situation est accentuée par la tendance, depuis déjà plus d'une décennie, à la désinstitutionalisation de la prise en charge psychiatrique, c'est-à-dire concrètement au raccourcissement des durées d'hospitalisation, ce qui impose de trouver des « relais » lorsque les patients ne sont pas insérés et/ou présentent manifestement quelques difficultés à l'être. En pratique, à l'issue de la prise en charge hospitalière en psychiatrie, ou d'une façon plus générale de la phase initiale d'assistance en matière de soins, les patients non insérés professionnellement se voient adressés à la COTOREP, pour établir s'il y a lieu leur statut de handicapé (on parle aujourd'hui de " handicap psychique », pour le distinguer du « handicap mental » d'ordre essentiellement déficitaire). Cette appréciation du handicap soulève immédiatement la question de l'aptitude au travail requise pour bénéficier du statut de «travailleur handicapé » (et par suite des « avantages » qui leur sont réservés), ou lorsque cette aptitude fait défaut, l'opportunité d'une allocation spécifique est évaluée par la deuxième commission de la COTOREP.

Lorsque l'appréciation d'aptitude au travail est problématique, ou que l'orientation vers un emploi effectif reste floue ou très incertaine, un bilan d'évaluation spécialisée peut être demandé, pour préciser les capacités de la personne et contribuer à son orientation effective. Pour les personnes dont le retour au travail en milieu ordinaire n'est pas immédiatement possible, il existe une variété de structures intermédiaires, susceptibles de proposer une activité adaptée aux possibilités de la personne, éventuellement de "préparer » au retour à l'emploi en milieu ordinaire. Dans le milieu protégé, en particulier les CAT, l'accueil de handicapés psychiques est une pratique relativement nouvelle et qui tend à se développer, aboutissant à un changement notable de la population d'usagers de certains de ces établissements.

Cette tendance actuelle à promouvoir la réinsertion sociale (et notamment professionnelle) des malades mentaux ne semble cependant pas simplement explicable par ces dispositions législatives. On l'observe en effet 
également, et de façon parfois plus marquée, dans d'autres pays, notamment de culture anglo-saxonne, dont le contexte politique est autre et où cette promotion de la réinsertion sociale s'avère essentiellement soutenue par le milieu associatif. Le courant de pensée qui soutient ces pratiques, sous le terme générique de "réhabilitation psychosociale ", est d'ailleurs issu et imprégné de ce contexte libéral et militant.

\section{ENTRE LE MONDE DES SOINS ET LE MONDE DU TRAVAIL,} LE SECTEUR MÉDICO-SOCIAL EST-IL UN MONDE DE TRANSITION?

Beaucoup de travaux sur ces pratiques d'aide à la réinsertion insistent avec raison sur le transfert, quant à la prise en charge de ces personnes, du secteur sanitaire au secteur médico-social, et les auteurs s'accordent en général à souligner la nécessité de promouvoir et d'améliorer l'articulation entre ces deux secteurs. Cette demande est en elle-même révélatrice de l'hétérogénéité et de la réelle séparation entre ces deux secteurs d'activité. Il nous paraît utile, pour commencer, de tenter de comprendre cette situation qui constitue l'arrière-plan ou le contexte de tout ce qui se fait en matière de réinsertion des handicapés.

En premier lieu, on peut chercher à préciser ce que recouvrent ces termes englobants de "secteur sanitaire » et "secteur médico-social », aussi bien d'ailleurs pour les « usagers » qui les traversent, que pour les «professionnels » qui y sont insérés. Si initialement ces secteurs sont découpés administrativement, notamment par leurs modes de financement (l'assurance maladie pour le sanitaire, l'état et les collectivités locales - les conseils généraux en particulier - pour le médico-social), et par leurs ministères de tutelle respectifs, la différence entre ces secteurs d'activité va bien plus loin. Presque tout les distingue ; en premier lieu leurs objectifs : les soins dans le premier cas, la réinsertion ou l'intégration sociale dans le second, mais aussi la formation des professionnels qui y travaillent : les soignants pour le sanitaire (médecins, infirmières, psychothérapeutes), les formateurs, éducateurs, assistants sociaux, gérants de tutelle pour le secteur médico-social. Or ce qui est associé à ces qualifications professionnelles, ce sont de véritables « cultures » propres à ces domaines d'activité. Le monde médical en est particulièrement représentatif, avec son vocabulaire technique propre, une division du travail très clairement spécifiée et un mode de coordination de ces fonctions rigoureusement hiérarchisé, héritier de la tradition hospitalière mais continuant d'infiltrer toutes les activités de soins. Ces dernières sont d'autre part régulées par une série de principes et d'exigences, et de cette façon véhiculent tout un système de valeurs. Dans cet univers, le patient, comme cette désignation l'indique, tend à être assigné à une position passive ; la pratique 
$\mathrm{du}$ soin reste celle du professionnel et exige une expertise. Du patient sont attendues sa coopération mais aussi sa docilité et sa confiance. On pourrait développer encore cette caractérisation du "monde sanitaire », mais cela suffit pour remarquer que cette « culture » médicale n'est pas celle du secteur médico-social. Sans doute y trouve-t-on aussi un vocabulaire technique probablement moins développé, mais il est clair que la division du travail n'y est pas spécifiée de la même manière, de même que la hiérarchisation des fonctions. Quant à ses objectifs, l'intégration sociale et l'autonomie, ils sont clairement distincts (mais pas contradictoires) des objectifs de soins, la guérison ou la stabilisation. Enfin la position de "l'usager » dans ces dispositifs est nécessairement plus active, et la tâche des professionnels est souvent définie en termes d'accompagnement. Ce qui apparaît ainsi comme une véritable différence de cultures (comportant chacune leur système de valeurs, leurs modes de coordination, leurs codes relationnels) entre le " monde sanitaire " et le " monde médico-social » permet notamment de comprendre, dans une certaine mesure, les conflits récurrents (ou du moins les tensions ou malentendus courants) survenant, au sein du secteur médico-social, entre les professionnels du soin qui y travaillent (médecins, infirmières) et les cadres ou le reste du personnel de ces établissements. L'articulation de ces mondes (celui des soins, celui de la réinsertion) ne va manifestement pas de soi. Cela apparaît nettement pour les professionnels, mais qu'en est-il pour les patients (ou usagers) qui fréquentent aussi, alternativement, l'un et l'autre de ces mondes?

Avant de revenir à cette question, il est intéressant de prolonger l'idée de considérer le secteur sanitaire et le secteur médico-social comme des microcultures hétérogènes, ce qui conduit à admettre que le monde du travail en constitue également une, comportant aussi son vocabulaire propre, ses exigences et ses valeurs spécifiques. Dès lors, on peut se demander dans quelle mesure on peut considérer qu'entre le monde des soins et le monde du travail le monde médico-social fonctionne comme un intermédiaire, un monde de transition ; c'est d'ailleurs une fonction que de nombreux travailleurs sociaux revendiquent. À beaucoup d'égard, cette idée apparaît juste : toute une activité du secteur médico-social vise à " acclimater » les " usagers » aux contraintes du monde du travail, voire à celles du monde urbain en général, tout en s'adaptant à leurs difficultés spécifiques.

Ce constat de l'hétérogénéité de ces mondes disjoints (monde du travail, monde des soins, monde médico-social), avec leurs cultures propres, nous paraît important car cette situation pose un ensemble de difficultés, mais aussi offre certaines ressources, à la fois aux « usagers » confrontés alternativement à ces divers mondes, mais aussi aux professionnels dont l'activité s'insère dans l'un ou l'autre de ces mondes et enfin, quoique de façon plus 
marginale, au «théoricien » en quête d'une construction théorique intégrée pour appréhender le parcours d'insertion professionnelle. Nous considérerons brièvement ces trois points de vue :

- pour le patient ou l'usager, on peut se demander comment il appréhende ces mondes distincts et quel type de représentation il a de leurs différences. Il est clair que la démarche d'insertion impose de circuler entre ces mondes culturels distincts, au point qu'on peut se demander si la faculté d'intégrer ces mondes sans les confondre et en sachant passer de l'un à l'autre ne constitue pas l'un des aspects importants de la « compétence sociale». On aurait cependant tort de croire que si l'articulation de ces « mondes » (des soins, du travail...) pose des problèmes aux praticiens (qui revendiquent une meilleure coordination entre eux) ou au théoricien (confronté à l'hétérogénéité des pratiques et des discours), elle en pose nécessairement plus aux usagers. Ce ne sont en tout cas pas les mêmes problèmes. L'usager n'a pas à comprendre les rapports complexes qu'entretiennent ces mondes, mais seulement à savoir s'y comporter, et passer d'un monde à l'autre n'est rien d'autre que de passer d'une activité à une autre. Il nous semble même au contraire que certaines personnes, en dépit de leurs difficultés psychiques, trouvent dans cette hétérogénéité du monde des soins et du monde du travail non des difficultés mais au contraire l'opportunité d'adopter de nouvelles attitudes, de s'installer dans une autre position ou fonction, profitant d'un contexte nouveau doté d'objectifs propres. Bref cette hétérogénéité des mondes pourrait bien jouer comme ressource pour les patients plutôt que comme difficulté à surmonter, pour certains d'entre eux au moins ;

- pour les professionnels concernés par ces pratiques d'accompagnement à la réinsertion, et en particulier pour les cliniciens, il est clair que leur pratique est fortement contextualisée par le secteur d'activité où elle s'insère. Ce n'est pas la même chose d'être clinicien dans une structure de soins ou dans une structure de soutien à la réinsertion. Il est vrai que le clinicien peut établir, dans une certaine mesure, un rapport distancié aux objectifs premiers du secteur d'activité où il intervient, mais c'est encore une façon de tenir compte de ces objectifs et de situer ses interventions par rapport à ceux-ci. Il revient donc à chaque professionnel, et tout particulièrement au clinicien, de trouver une position juste dans le secteur d'activité où il est inséré, contribuant à l'activité (c'est-à-dire aux objectifs premiers) de ce secteur, tout en sachant tenir compte en pratique des objectifs de l'autre secteur d'activité. Cela le conduit à se soucier de la coordination entre ces mondes. L'enjeu de cet ajustement du clinicien est que le décalage propre à la posture clinique soit perçu, relativement à l'activité de l'institution, comme un enrichissement, un apport complémentaire, et non comme une perspective discordante et inintégrable. 
(Nous avons indiqué le fréquent décalage entre l'approche clinique, influencée par le monde des soins, et les activités du secteur médico-social) ; - pour le théoricien enfin, en quête d'une construction théorique intégrée en matière d'insertion des handicapés psychiques, l'hétérogénéité des pratiques et des discours du secteur sanitaire et du secteur social se présente d'abord comme une difficulté. Cette difficulté est en fait révélatrice de la complexité de ce qui est en cause, de son caractère pluridimensionnel, de la variété des perspectives possibles, et finalement de la richesse de ce domaine de recherche si on prend le temps d'en explorer les composantes.

L'hétérogénéité culturelle des mondes en question (monde des soins, monde du travail), se manifeste d'emblée dans les divergences terminologiques, à commencer par le mode de désignation du bénéficiaire de ces pratiques. De «patient » dans le monde médical, il devient «l'usager » ou le « travailleur handicapé » dans le secteur médico-social, puis «l'employé » ou « le salarié » dans le monde du travail. Ces différences terminologiques ne sont pas accidentelles. Elles traduisent des différences considérables de position, de fonction ou de rôle social. On retrouve un écart analogue entre les projets élaborés à chacun de ces niveaux. Le " projet thérapeutique » du monde médical est remplacé dans le secteur médico-social par le « projet d'insertion », auquel suivra dans le monde du travail le " plan de carrière ». Ces différences de terminologie qui traduisent des déplacements de perspective et des changements d'objectif posent un problème au théoricien qui voudrait développer une vision englobante. A-t-il affaire à un patient, à un usager, à un travailleur ? Que signifie ce changement de statut selon les étapes du parcours ? De quel type de projet est-il question et comment ces divers types de projets s'articulent-ils entre eux ? Faut-il respecter la pluralité des perspectives et admettre une irréductible hétérogénéité entre la théorisation des soins et celle de l'insertion au travail ?

Au-delà de ce constat d'hétérogénéité et de disjonction entre le monde des soins, le monde du travail, et ce monde de transition que propose le secteur médico-social, on peut d'un point de vue théorique se demander s'il faut se résoudre à cette disjonction et en tirer les conséquences, aller pour ainsi dire dans son sens, en privilégiant une partition du travail, en revendiquant l'intérêt de disjoindre l'activité de soin et l'activité de réinsertion (et leurs théorisations respectives), ou s'il est préférable au contraire de défendre une conception plus intégrée des soins et de la prise en charge sociale, où le parcours d'insertion serait considéré comme en continuité avec la démarche de soins, où en pratique les mêmes équipes assureraient les deux fonctions...

Remarquons d'abord que si cette question est légitime d'un point de vue théorique, il ne faut pas se faire trop d'illusions sur l'incidence de ces réflexions sur les pratiques, ces dernières étant surdéterminées par des choix 
politico-administratifs, à commencer par les dispositions législatives qui, en décidant des modes de financement, structurent les modes de prise en charge. Il est vrai que ce type de contraintes déterminantes est pondéré par $\mathrm{d}^{\prime}$ autres dynamiques lentes, telles que des logiques institutionnelles (qui à la tendance actuelle à une partition des tâches opposent la tendance institutionnelle à une prise en charge durable et tendanciellement totalisante faute, il est vrai, de relais...) Bref, sans ignorer que les pratiques restent très dépendantes de facteurs sur lesquels les arguments théoriques ont peu de prises, on peut cependant comparer les options et prendre position.

Nous commencerons par reconnaître qu'il y a des arguments pour défendre l'une ou l'autre perspective. Brièvement, nous exposerons les arguments qui nous conduisent à estimer préférable une approche où restent bien distinctes l'activité de soins d'une part et les pratiques médico-sociales de soutien à la réinsertion de l'autre. Le principal argument est tout simplement la profonde différence des objectifs qui définissent et structurent ces pratiques et en font des activités complètement différentes, ce qui ne signifie pas inconciliables. Pour la même raison, à savoir la spécificité et l'hétérogénéité de ces activités, il nous paraît préférable d'en développer des théorisations distinctes, attentives justement à la spécificité de ce qui est en cause, par exemple pour la réinsertion à la spécificité de l'activité de travail et de ce qu'elle implique pour le sujet qui s'y engage. Comme on le verra, le soutien à l'insertion est une pratique déjà hybride, qui risque de perdre en pertinence et en efficacité si elle doit comporter en plus le souci du soin. On peut en dire de même de la théorisation qui est à développer en suivant le fil de ce qui est spécifique dans ces pratiques. Si cette théorisation n'est qu'une extension de la théorisation des soins, elle a beaucoup moins de chances de saisir le propre de la problématique de l'insertion dans le travail.

Ajoutons que cette pratique de soutien et d'accompagnement à l'insertion est une pratique relativement récente, qui gagnerait à trouver son style propre, sa théorisation spécifique, en s'émancipant du modèle du soin qui, tant qu'il reste présent, surplombe nécessairement les efforts de théorisation. Enfin sur un plan à la fois pragmatique et politique, relativement à la place sociale faite aux malades et handicapés, le désengagement relatif mais tendanciel du secteur sanitaire dans la prise en charge des malades mentaux ne laisse tout simplement guère d'autre alternative que le développement de ce type de structures associatives, dont la fonction prioritaire est justement d'assurer ce relais, après l'étape des soins, en visant à promouvoir l'intégration sociale. 


\section{LE BILAN D’ÉVALUATION SPÉCIALISÉE : UNE ACTIVITÉ PLURIELLE}

Le bilan d'évaluation peut être simplement défini par ses objectifs : il vise à apprécier l'aptitude au travail de personnes qui ont présenté des troubles psychiatriques et qui ne sont pas, ou plus, insérées professionnellement. Il s'agit de personnes qui ne font plus l'objet de soins quotidiens en milieu hospitalier (ou hôpital de jour), et pour lesquelles doivent être envisagées par conséquent les modalités de leur réinsertion sociale. Se pose en particulier la question de leurs moyens de subsistance, celle des revenus et par conséquent celle des possibilités de réinsertion professionnelle ou, à défaut, de recours aux allocations spécifiques. Quant au retour au travail, il peut être envisagé soit dans le type d'emploi exercé auparavant, soit dans le cadre d'un reclassement professionnel, ce qui suppose très souvent la nécessité d'une nouvelle formation. Pour les personnes sans formation professionnelle antérieure, se pose également la question d'une formation et par conséquent au préalable celle de leur orientation et du choix d'une activité adaptée, puis éventuellement du type de formation qui y donne accès. Pour les personnes enfin dont les possibilités d'adaptation au travail en milieu ordinaire apparaissent très incertaines, peut être également proposé un emploi en milieu protégé, de type CAT ou atelier protégé ; comme pour la réorientation professionnelle, le choix d'une activité adaptée aux compétences et intérêts de la personne doit être précisé, en tenant compte des possibilités offertes par ces structures et des intérêts et aptitudes de la personne concernée. Quant aux personnes qui n'apparaissent pas immédiatement en mesure de s'adapter à un contexte de travail mais dont l'état est susceptible d'évoluer pour rendre envisageable un tel projet, des structures de préparation à l'emploi peuvent être proposées, qu'il s'agisse d'ateliers thérapeutiques (ou sociothérapeutiques) dont le fonctionnement s'apparente à celui des hôpitaux de jour mais avec le souci et l'objectif de préparer à un retour au travail, ou de stages de préorientation lorsque reste surtout à préciser un choix d'orientation professionnelle adapté. Lorsqu'enfin un projet d'insertion professionnelle n'apparaît pas envisageable, à relativement court terme, parce que les troubles résiduels restent encore trop invalidants ou parce $\mathrm{qu}^{\prime}$ un tel projet d'insertion ne correspond pas au souhait de la personne, sont essentiellement préconisés la poursuite (ou la reprise) des soins et le recours à d'autres modes d'insertion sociale.

En pratique et pour chaque personne, ce qui est attendu du bilan est précisé par les prescripteurs de cette prestation. Ceux-ci appartiennent aux organismes chargés $d^{\prime}$ insertion et/ou de statuer sur l'aptitude au travail : principalement la « COTOREP » et les « cap-emploi ». Dans le premier cas, le bilan constitue une investigation complémentaire destinée à évaluer l'apti- 
tude à l'emploi (en milieu ordinaire ou protégé), et éventuellement aussi l'opportunité d'une formation et la nature de celle-ci. Dans le cas des « capemploi ", il s'agit d'organismes destinés à l'insertion de personnes souffrant de handicaps de toutes natures (physique, sensorielle, psychique) et en difficulté d'insertion. Le personnel de ces structures n'ayant pas de formation spécifique sur le plan médical ou psychopathologique, le bilan constitue dans ce cas un « appui technique » destiné à éclairer le chargé d'insertion sur les possibilités et les limites de la personne, en tenant compte de sa formation et de son expérience professionnelle antérieure, mais aussi et surtout de ses antécédents psychopathologiques et de ses difficultés résiduelles sur ce plan.

Ces bilans, initialement et encore partiellement financés par l'AGEFIPH, sont pour l'instant réservés aux personnes bénéficiant déjà du statut de travailleur handicapé. On peut enfin caractériser sommairement les bénéficiaires de cette prestation en indiquant qu'il s'agit d'adultes jeunes, pour la majorité entre 25 et 45 ans (mais certains plus âgés), et dont le trouble le plus fréquemment observé est de nature psychotique, les autres situations psychopathologiques se répartissant entre troubles bipolaires, états limites, troubles invalidants de nature névrotique (TOC) et somatisations, troubles de la personnalité enfin, que nous qualifierons de socialement invalidants. Si les conduites addictives ne sont pas exceptionnelles, il est rare qu'elles nous apparaissent devoir être retenues comme le diagnostic principal.

Ces bilans qui, dans leurs conclusions, doivent argumenter un jugement d'aptitude au travail, et autant que possible indiquer les types de soutien pouvant être apportés à la démarche de réinsertion, supposent d'apprécier non seulement les compétences instrumentales de la personne (formation antérieure, compétences professionnelles ou techniques), mais aussi la capacité de la personne à s'adapter au «monde du travail » et à ses exigences (horaires, intégration dans une équipe, rythme de travail, productivité...). Ce jugement d'aptitude se fonde, pour une large part, sur l'appréciation des troubles psychopathologiques résiduels et leur retentissement sur les performances cognitives et les facultés relationnelles des patients. Nous présenterons brièvement les modalités de notre pratique de bilan puis évoquerons les dimensions qui nous paraissent pertinentes pour étayer cette appréciation du potentiel de réinsertion et des moyens d'apporter un soutien à cette démarche.

\section{1 Premières remarques tirées d'une expérience de la pratique de bilans}

Pour évoquer très brièvement notre expérience de la pratique de bilan, nous nous limiterons à mentionner les outils d'observation et d'évaluation auxquels nous avons recours, et les principales dimensions qui nous sem- 
blent pertinentes pour étayer ce pronostic d'aptitude au travail (et éventuellement les conseils d'orientation). Ces bilans sont toujours réalisés par une équipe impliquant au minimum trois intervenants : un psychologue chargé du bilan, un psychologue chargé de la passation et de l'interprétation des tests psychotechniques ainsi que de la restitution des résultats à la personne évaluée, et un psychiatre chargé plus spécifiquement de l'évaluation psychopathologique. Sur le plan technique, les situations d'observation sont les entretiens cliniques, les tests psychotechniques visant à apprécier le niveau de compétence scolaire et les facultés de raisonnement et d'apprentissage, et deux demi-journées de travail en groupe, qui permettent d'apprécier les capacités de la personne à établir un contact avec les autres et à s'adapter à cette situation de groupe aussi bien qu'à des situations nouvelles.

Nous vérifions pour ainsi dire quotidiennement l'intérêt de croiser ces modes d'observation, ainsi d'ailleurs que nos points de vue cliniques distincts, les personnes laissant souvent apparaître des facettes différentes de leur personnalité et de leur problématique selon les contextes d'investigation et les interlocuteurs.

Nous n'aurons pas besoin d'insister ici sur l'importance des entretiens permettant que la prestation ne se limite pas à une pure évaluation, mais comporte une dimension d'interaction, avec ce que la rencontre peut produire d'inattendu, avec la possibilité aussi d'apporter un étayage à ces consultants fragilisés psychologiquement. Il faut à ce propos souligner que ces bilans d'évaluation peuvent représenter, pour les personnes concernées, des enjeux allant bien au-delà d'une simple expertise technique dans leur parcours de réinsertion. Certains voient dans cette appréciation de leur potentiel d'insertion un véritable pronostic formulé à partir de la forme, plus ou moins stabilisée, qu'a pris leur maladie. La possibilité de réinsertion professionnelle est souvent vécue comme un gage de "normalité » sociale et en ce sens de "guérison ", ce qui peut bien sûr contribuer à soutenir la motivation à la réinsertion professionnelle, mais peut aussi dans certains cas rigidifier la décision d'une personne de retrouver un emploi en milieu ordinaire (voire tel emploi défini), sans accepter les phases préparatoires pourtant requises. Enfin, l'échange autour des résultats et des éléments observés, au cours et en fin de bilan, permet non seulement d'optimiser la qualité de la relation et par conséquent aussi la qualité des informations recueillies, mais aussi d'apprécier l'aptitude des consultants à intégrer ces éléments, ce qui est un indice de souplesse adaptative. L'échange que constitue l'entretien permet aussi d'aider à l'élaboration du projet d'insertion qui, bien souvent, sera à poursuivre avec les organismes d'insertion. 
4. 2 Quelques dimensions pertinentes pour évaluer l'aptitude à l'emploi et éclairer l'orientation professionnelle

4. 2. 1 Le mode de stabilisation des troubles et leur incidence sur les capacités (1) professionnelles et (2) d'insertion

Il relève pour ainsi dire du bon sens que le potentiel d'insertion dépende, dans une large mesure, de la nature des troubles psychopathologiques présentés et de leur mode de stabilisation. Cela ne doit cependant pas conduire à sous-estimer l'importance d'autres facteurs comme le niveau de formation initialement atteint ou l'expérience professionnelle acquise antérieurement aux troubles, dont certaines études récentes soulignent l'importance comme facteur de réinsertion (McGurk et al., 2000). Il reste donc à préciser le rôle que joue, dans l'évaluation, l'appréciation de la nature de la pathologie, même quand cette dernière peut être considérée comme stabilisée. Les pathologies mentales se distinguent en effet des pathologies somatiques par le fait qu'il ne s'agit pas seulement d'apprécier les séquelles qu'elles ont laissées, mais leur mode de stabilisation et leur profil évolutif prévisible. S'il est vrai que le diagnostic ne peut suffire à faire un pronostic d'aptitude au travail, il importe à notre sens pour apprécier au moins deux types de facteurs conditionnant la réinsertion :

1. Le degré de stabilisation, l'importance des troubles résiduels et le profil évolutif envisageable.

2. Le retentissement des troubles (résiduels) sur un ensemble de dimensions qui, elles, sont directement pertinentes pour l'aptitude au travail et l'orientation.

Précisons rapidement ces deux facteurs.

1. Le type de stabilisation et le mode d'évolution envisageable de la pathologie

L'évaluation du type de stabilisation relève d'une appréciation clinique, c'est-à-dire pluridimensionnelle, s'efforçant d'appréhender non seulement les troubles résiduels décelables, mais aussi leur retentissement fonctionnel sur les principaux secteurs de l'existence du sujet, en s'attachant à saisir diachroniquement les modes de manifestation et l'incidence de ces troubles.

Quant aux modalités d'évolution des troubles, on tente de les apprécier à partir de la nature des troubles (c'est-à-dire du diagnostic) et des connaissances théoriques et pratiques disponibles sur les modalités évolutives prévisibles de cette pathologie. Un indicateur également précieux est le profit évolutif des troubles déjà observable chez la personne concernée, donc l'histoire de sa maladie, et en particulier l'ancienneté d'apparition des troubles et leur évolution passée. 
On peut schématiser les principales configurations évolutives rencontrées de la façon suivante : 1) Stabilisation après amendement des troubles ; il ne persiste qu'une fragilité et/ou un risque de rechute ;2) Persistance des troubles dont l'intensité est à apprécier, avec une stabilisation sur un mode chronique ; 3) Alternance de périodes d'amélioration et de rechutes, dont il importe de repérer les facteurs déclenchants quand ils existent et peuvent être éventuellement évités. Il est clair qu'en l'absence de stabilisation, il est encore plus difficile de pronostiquer l'aptitude à l'emploi. À l'inverse, une stabilisation déjà ancienne avec une reprise d'activité depuis cette stabilisation est un indice de bon pronostic pour l'insertion.

\section{2a. Interférence des troubles sur les capacités professionnelles}

Il est clair que les troubles retentissant directement sur les facultés d'attention et de concentration ont une incidence sur les performances professionnelles. Ce peut être le cas par exemple des symptômes psychotiques productifs (hallucinations, préoccupations délirantes), mais aussi de certains symptômes anxieux ou névrotiques tels que les troubles obsessionnels compulsifs ou les états d'inhibition, qu'ils soient de nature anxieuse ou liés à un ralentissement dépressif important. Ont également une incidence directe sur les performances au travail (envisagées cette fois de façon globale), les symptômes psychotiques déficitaires (apragmatisme, perte des initiatives, désintérêt et désinvestissement, repli et passivité), mais aussi les troubles dépressifs sévères par le biais du ralentissement et du doute de soi. Quant au retentissement sur le potentiel intellectuel et les capacités d'apprentissage, on l'apprécie à l'aide des tests psychotechniques qui ne permettent cependant guère de distinguer ce qui relève du niveau de compétence acquis antérieurement de ce qui relève de l'incidence de la maladie sur cette compétence antérieure.

\section{2b. Interférence des troubles sur les capacités d'insertion}

Il s'agit principalement des facultés d'adaptation aux contraintes propres au monde du travail (contraintes de déplacement, d'horaires, de rythme, d'organisation impliquant une « hiérarchie »...), et nous mettrons à part l'adaptabilité relationnelle, facteur indéniablement important pour une insertion professionnelle durable et relativement indépendant des compétences techniques à occuper un poste. En pratique, cette souplesse adaptative apparaît souvent cliniquement corrélée à la reconnaissance, par la personne, de ses troubles ou limites. Cela constitue un indice de bon pronostic pour la réinsertion professionnelle et sociale, pour des raisons sur lesquelles nous allons revenir. Il s'avère finalement important qu'un travail psychique d'élaboration et d'acceptation du handicap ait eu lieu pour que la personne puisse réellement investir un projet de réinsertion. 


\section{2. 2 Les composantes de la motivation et l'incidence de l'image de soi}

Qu'il s'agisse des démarches de réinsertion en général, ou spécifiquement du bilan d'évaluation, on suppose toujours de la part de l'intéressé une demande, qui mérite cependant d'être précisée. Elle traduit notamment les attentes de l'intéressé et sa représentation de la situation, en particulier de lui-même et de l'activité qu'il envisage. Elle contribue à l'appréciation de sa motivation et de ce qui est à l'origine de ses démarches. Il s'avère parfois en effet que cette demande émane de l'entourage. Il convient alors d'apprécier le degré d'adhésion du consultant à ce projet, éventuellement l'appropriation qu'il a pu en réaliser, ou s'il ne fait que s'y soumettre de façon passive.

Une authentique motivation est souvent soutenue par des possibilités dont l'emploi est la condition, par exemple le désir d'accéder à plus d'autonomie en pouvant disposer d'un logement et de revenus propres, ou un souci d'amélioration des revenus, ou encore un souhait d'insertion sociale et d'intégration, en particulier chez les patients sensibles à l'identité sociale que confère un emploi. Dans certains cas enfin, l'emploi est considéré comme un gage de guérison, la discipline de vie qu'il impose pouvant apparaître sécurisante. Inversement, la motivation peut être incertaine, ambivalente, ou absente comme dans les cas de soumission passive à l'incitation d'un tiers (parents, médecin, assistante sociale...) ou quand existe une hésitation à renoncer à une situation de dépendance vécue comme avantageuse, qu'il s'agisse de dépendance matérielle ou affective.

La notion même de motivation, si elle renvoie indiscutablement à une dimension déterminante pour l'insertion, est en elle-même floue et requiert des distinctions. Ce n'est clairement pas la même chose d'être motivé par le désir d'atteindre un objectif sans connaissance précise des moyens d'y parvenir (et en particulier de ses possibilités propres à atteindre ce but), ou d'être motivé justement par la connaissance effective de ses aptitudes et compétences (fondée sur des expériences antérieures) et par la conviction dérivée que l'objectif est non seulement à la portée du sujet mais ne requiert qu'un effort prévisible. Dans le premier cas, la motivation est liée simplement à un imaginaire associé à un désir lui-même éventuellement vague, alors que dans le second, elle est fondée sur l'appréciation de ses propres aptitudes et possibilités, et sur le désir d'en faire usage. Le facteur discriminant relève de l'autoévaluation de ses aptitudes et par conséquent de facultés métacognitives (c'est-à-dire d'appréciation et de contrôle de ses propres ressources cognitives), facultés qui justement apparaissent altérées dans nombre de situations psychopathologiques (pour des raisons qui seraient à préciser). En matière d'activité professionnelle, cette auto-appréciation de ses compétences dépend évidemment des expériences de travail antérieures, et en particulier 
des tâches réalisées avec succès. C'est sur ce type d'expériences antérieures positives, qui justement font défaut aux sujets désinsérés de longue date, que se construit non seulement l'appréciation de sa compétence, mais ce que Bandura appelle " le sentiment d'efficacité personnelle » qui s'avère un des déterminants de la motivation à entreprendre, de la confiance en soi, et finalement un des composants majeurs de l'image de soi.

L'intérêt, en particulier dans cette problématique de l'insertion professionnelle, de ce concept d'image de soi tient en premier lieu à l'altération de cette dimension par la maladie mentale (soit directement, soit indirectement par l'image sociale négative associée à ces pathologies, soit enfin par la désinsertion sociale provoquée par la maladie.) Il importe également de remarquer les conséquences invalidantes de cette altération de l'image de soi (déficit de confiance en soi, perte d'initiative, anticipations négatives renforçant les inhibitions...). L'importance de ce concept d'image de soi nous paraît sousestimée dans le domaine qui nous occupe. La représentation que les individus ont d'eux-mêmes (ce qu'ils pensent, ressentent ou croient d'eux-mêmes) s'avère en effet un des régulateurs les plus puissants de beaucoup de leurs comportements et une des sources de leur motivation (Gecas, 1991). S'il en est ainsi, cette dimension d'image de soi ne peut avoir qu'une incidence majeure sur l'aptitude à se réinsérer professionnellement, et par conséquent devrait faire l'objet d'une attention particulière dans nos pratiques d'évaluation.

L'intérêt que portent classiquement les cliniciens à " la conscience des troubles » (cf. § 4. 2. 1), et leur tendance à y voir un indicateur des possibilités d'adaptation et donc du pronostic quant à l'insertion, trouve sans doute là une explication, la conscience des troubles intervenant comme un indicateur d'une faculté métacognitive plus générale, dont dépend l'adaptation sociale et l'intégration au travail.

L'approfondissement théorique de cette dimension complexe mais importante qu'est la motivation, ainsi que de ses déterminants, serait à développer dans le cadre des recherches empiriques et théoriques actuelles ayant trait aux facultés métacognitives (Proust, 2004), et à leur contribution à ce qu'on rassemble sous le terme « d'image de soi », ou encore plus globalement de « conscience de soi ».

\section{3 Activités inhérentes au bilan de réinsertion et horizons corrélatifs de théorisation}

Si on cherche à poursuivre l'analyse de ce type de pratique de bilan, et à l'étayer par des repères et hypothèses théoriques, il importe de commencer par reconnaître que cette pratique recouvre en réalité une pluralité d'activités qui renvoient chacune à un domaine de compétence et à des arrière-plans 
théoriques propres. Schématiquement, on peut spécifier ces activités de la façon suivante, en les rattachant à des pratiques d'évaluation psychologique déjà bien établies :

- l'évaluation psychopathologique : nous venons d'insister sur le fait que le jugement d'aptitude au travail et l'appréciation pronostique quant à l'adaptabilité sociale (au contexte de travail) (de même que la proposition d'éventuelles étapes préparatoires au retour à l'emploi) reposent en grande partie sur une évaluation psychopathologique, que nous ne développerons pas plus ici ;

- le bilan de compétence : essentiellement destiné à spécifier les possibilités d'insertion professionnelle, en fonction des compétences professionnelles acquises antérieurement, du niveau global de formation (évaluation des acquis scolaires), en tenant compte de la singularité de la personne, en l'occurrence de ses souhaits d'orientation mais aussi de ses difficultés psychiques, le bilan s'apparente à un bilan de compétence, et pourrait sans doute bénéficier des recherches et hypothèses théoriques développées autour de cette pratique ;

- le conseil d'orientation: comme nous l'avons indiqué, dans un grand nombre de cas où le retour à l'emploi s'avère envisageable, la tâche principale reste de préciser une orientation professionnelle adaptée aux préférences de l'intéressé quant au type d'activité professionnelle, mais aussi à ses compétences et éventuellement à ses limites liées à sa vulnérabilité psychopathologique. Cette tâche s'avère d'autant plus importante que nombre de personnes, tout en revendiquant leur motivation à se réinsérer professionnellement, n'ont étonnamment pas d'idée d'orientations possibles. La première étape de l'élaboration du projet d'insertion s'apparente donc à du conseil d'orientation professionnelle. Or ce type d'activité a fait l'objet de recherches et d'élaborations théoriques dont on gagnerait également à s'inspirer. En particulier, la fonction singulière de conseiller d'orientation a fait l'objet d'analyses fines, faisant apparaître la superposition d'activités (voire de rôles) condensées dans cette fonction, chacune de ces activités renvoyant à des arrière-plans théoriques et philosophiques propres (Blanchard, 2000, 2003).

L'étayage théorique de la pratique de bilan pourrait donc commencer par chercher à exploiter le legs des recherches et élaborations théoriques développées à partir de ces pratiques connexes et mieux instituées. Il est tout simplement dommage de ne pas tirer profit de l'expérience acquise et des recherches faites sur des activités apparentées. Il ne s'agit cependant pas simplement d'importer dans le contexte psychopathologique ces acquis ou ces problématisations, mais d'en apprécier la pertinence dans le cas du handicap psychique, de les adapter éventuellement et d'en inférer de nouvelles hypothèses et axes de recherche, propres au contexte psychopathologique. Ce genre de confrontation interdisciplinaire ne peut manquer d'intérêt pour un 
champ d'activité dont le développement est récent et sur lequel la réflexion théorique débute.

Avec le modeste recul de notre expérience, il nous apparaît que la difficulté spécifique à cette pratique de bilan de réinsertion est non seulement celle d'apprécier la diversité des facteurs susceptibles d'influer sur la réinsertion, mais celle d'apprécier pour chaque cas la pondération de ces facteurs, le poids respectif de leur influence sur le potentiel de réinsertion. Nous avons insisté sur la nécessité d'une évaluation psychopathologique, qui fournit en effet des repères indispensables mais en pratique jamais suffisants à apprécier les possibilités d'insertion. Il est vrai que dans certains cas, ces facteurs psychopathologiques apparaissent déterminants (en particulier sur un mode négatif, quand ils spécifient les obstacles parfois difficilement surmontables à l'insertion), dans d'autres cas l'authenticité et la force de la motivation à la réinsertion font de cette dernière le facteur décisif de la réinsertion, reléguant à l'arrière-plan l'incidence des difficultés psychiques de la personne. Dans d'autres cas encore, l'expérience professionnelle acquise et la satisfaction à exercer telle activité constituent le socle d'étayage de la réinsertion, par le soutien de la motivation mais aussi la connaissance préalable du milieu pourvoyeur d'emploi. Dans d'autres cas enfin, le soutien apporté par "l'environnement », parfois les possibilités d'insertion offertes dans ce cadre (recrutement dans une entreprise familiale par exemple) contribuent évidemment efficacement à la réinsertion (du moins professionnelle).

Toute la difficulté mais aussi l'intérêt de cette appréciation du potentiel de réinsertion est de parvenir à appréhender cette pluralité de facteurs, dont la configuration est singulière à chaque cas, en estimant l'incidence respective probable de ces facteurs sur le parcours de réinsertion. Une manière $d^{\prime} y$ parvenir est de prêter prioritairement attention à la dynamique psychique dans laquelle s'inscrit la demande de réinsertion. Se rattache-t-elle à des enjeux psychiques essentiels ou se trouve-t-elle au contraire prise, voire empêtrée, dans des conflits psychiques non résolus et plus ou moins aigus ? Quelles significations symboliques peuvent se trouver associées à cette réinsertion (équivalence de guérison, d'accès à la maturité, à l'autonomie et en ce sens à la liberté...) et ainsi susceptible d'en faciliter ou d'en entraver le cours ? Quelles résistances enfin, éventuellement non conscientes, peuvent faire obstacle à la démarche d'insertion ?... Il est clair que sur ce plan, une approche $d$ 'inspiration psychanalytique trouve sa pertinence et peut aider à trouver un fil conducteur pour sélectionner et articuler les dimensions pertinentes. Observons enfin que l'appréhension de cette pluridimensionnalité sur laquelle nous mettons l'accent, ou si l'on préfère de la complexité de chaque situation, est après tout le propre de la pratique clinique. 
4. 4 Les difficultés et les ressources spécifiques, pour les handicapés psychiques, de l'intégration professionnelle

Nous souhaitons pour conclure esquisser une voie de recherche que nous croyons prometteuse, comme nous l'indiquons dans notre introduction. Il s'agit de tenter de comprendre d'abord ce qui se joue dans l'insertion professionnelle, dans l'engagement dans le travail, pour se demander seulement ensuite et sur cette base ce qui peut faire difficulté aux personnes souffrant de troubles psychopathologiques, dans leur rapport au travail. Autrement dit, le projet est de chercher à repérer ces possibles difficultés ou points $d$ 'achoppement en nous tournant non pas vers la vie psychique des personnes, en particulier vers leurs dysfonctionnements observables ou supposés, mais en nous tournant au contraire "du côté du travail ", pour considérer ce qui est propre aux situations de travail, les propriétés spécifiques à ce type particulier d'activité, et nous demander ensuite en quoi ces dernières pourraient constituer les points $d^{\prime}$ achoppement pour les personnes psychiquement vulnérables et en situation d'échec ou de désinsertion professionnelle. On peut en effet supposer que ces propriétés inhérentes au travail requièrent, de la part de celui qui assure le travail, des aptitudes ou plus simplement l'effectuation de tâches mentales, d'ajustement, de compromis, de coordination, de résolution de problème pas exemple, qui peuvent s'avérer difficiles aux personnes présentant une vulnérabilité psychique. En résumé et pour schématiser, il s'agit de chercher à comprendre ce qui fait difficulté non pas du côté du sujet mais du côté de la tâche et du contexte où elle doit être accomplie.

Pour simplifier et rendre plus précis cette réflexion, nous la restreindrons ici au cas des personnes présentant des troubles de nature psychotique, en particulier aux schizophrènes, ces personnes constituant la classe la plus représentative de celles qui nous sont adressées pour bilan.

La démarche que nous proposons est facilitée, et à vrai dire inspirée, par l'existence d'un corpus théorique riche en matière d'analyse du travail. Ces résultats, ceux en particulier qui nous semblent éclairants et sources d'hypothèses quant aux difficultés des psychotiques, résultent d'importants courants de recherche en psychologie du travail, inspirés notamment par la psychologie soviétique (Vygotski, Leontiev, Bakhtine). Nous ne ferons qu'esquisser cet axe de recherche en évoquant simplement des hypothèses que suggèrent certaines des propriétés du travail, dégagées et présentées par Y. Clot dans son ouvrage sur La fonction psychologique du travail (1999). Ces propriétés (et les aptitudes qu'elles sollicitent) peuvent nous mettre sur la voie de ce qui fait difficulté au patient psychotique pour s'intégrer au travail, mais elles peuvent aussi, de façon positive cette fois, indiquer ce que le tra- 
vail peut apporter de fondamental à ces patients, s'ils parviennent à s'y tenir, en particulier en matière d'intégration sociale.

D'entrée de jeu, et dans le cas spécifique de la schizophrénie, il y a toutes les raisons de s'attendre à des articulations pertinentes, mais qui restent à spécifier, entre une analyse du travail qui s'attache à préciser les activités mises en jeu, ainsi que le contexte de leur réalisation, et les théories contemporaines de la schizophrénie qui y voient essentiellement une pathologie de l'action, avec des troubles de l'initiation, de la coordination, de la représentation et de la conscience de l'action, corrélés à des dysfonctionnements cérébraux sur lesquels se concentre actuellement la recherche (Frith, 1992, Frith et al., 2000 ; Daprati et al., 1997 ; Proust, 2004). Le concept d'activité, comme schème intégrateur de divers plans d'action, peut sans doute contribuer à concevoir le lien, à notre avis encore très peu documenté, entre les troubles élémentaires de l'action étudiés chez les schizophrènes en situation expérimentale, et les conséquences de ces troubles à une échelle d'observation beaucoup plus intégrative, celle de la vie courante par exemple, ou justement celle des situations de travail qui sollicitent de façon particulière la mise en œuvre d'activités.

Il se dégage en particulier de l'analyse d'activités professionnelles ce qu'on pourrait appeler une problématique de l'appropriation, l'opérateur ou l'agent devant s'approprier la tâche qu'il a à accomplir, et qui pour ainsi dire «le précède », lui est initialement extérieure et même étrangère. D'autre part, cette tâche s'insère toujours dans un réseau d'autres tâches, souvent assurées par d'autres, et dont dépend son sens. De cette façon, toute tâche professionnelle s'inscrit, directement ou indirectement, dans une activité collective. Pour cette raison aussi elle est articulée à différents contextes, celui de son effectuation n'étant pas nécessairement celui de sa finalité ou de ce qui lui donne sens. Assurer une telle tâche suppose donc l'aptitude à s'approprier une intention initialement étrangère, mais aussi celle de comprendre comment s'insère cette activité dans le réseau d'activités dont elle est solidaire et dépendante, comment elle s'articule à différents contextes dont elles dépend. Or ces aptitudes, qui peuvent paraître banales et peu problématiques, sont précisément celles réputées déficientes chez les patients schizophrènes (Proust, 2000, 2004 ; Hardy-Baylé et al., 2003).

En s'appuyant sur l'analyse d'une tâche aussi complexe et exigeante que celle d'un pilote de ligne (tâche finement analysée pour comprendre les causes d'un accident aérien), Y. Clot va jusqu'à dire que " l'action se forme dans un milieu saturé d'activités hétérogènes en s'affranchissant - au mieux - de ses contradictions. Ce milieu... est son milieu intérieur en quelque sorte. Il est peuplé d'intentions étrangères que le professionnel doit se soumettre... Au bout du compte, l'action ne peut pas être comprise à par- 
tir d'elle-même. Son déclenchement doit être rattaché à des activités qui s'échangent dans certains contextes et son fonctionnement à des opérations qui s'exercent dans d'autres » (p. 26). Cette complexité (pluralité de contextes et de paramètres à coordonner) et cette hétéronomie de la tâche est sans doute maximisée dans le cas de cette activité hautement technique qu'est le pilotage, mais ces contraintes valent beaucoup plus largement, et s'exercent dans une moindre mesure sur toute activité professionnelle. Dans ce cas, l'exigence d'appropriation de la tâche, au sens d'en assumer la réalisation et la responsabilité, se traduit par l'exigence de "se déterminer », de décider et d'agir dans un milieu " saturé d'intentions étrangères », or cette détermination de l'action sur fond d'intentions étrangères (ou éventuellement cette appropriation d'intentions initialement étrangères) n'est-elle pas précisément ce qui peut être hautement problématique pour des schizophrènes qui, en phase de décompensation du moins, échouent à s'attribuer l'intention de leurs propres actions ou pensées spontanées. Les théories cognitives et neuroscientifiques contemporaines de la schizophrénie mettent justement l'accent sur les troubles de l'attribution, et plus généralement de la gestion des intentions dans cette pathologie (Frith, 1992 ; Campbell, 1999 ; Gallagher, 2000 ; Sarfati et al., 1999).

On peut faire le même type de remarque à propos de la pluralité et de l'hétérogénéité des contextes requis pour comprendre les facteurs de déclenchement de l'action d'une part et les modalités de son déroulement d'autre part. Or cette gestion complexe d'une pluralité de contextes hétérogènes, dont il convient pour agir de tenir compte sans les confondre, est précisément aussi une aptitude démontrée défaillante chez les schizophrènes (Hardy-Baylé et al., 2003).

Cette exigence de coordination de contextes hétérogènes, et même de domaines d'activités, dont il y a des raisons de penser qu'elle peut poser problème aux schizophrènes, serait pourtant une contrainte inévitable à toute activité professionnelle s'il est vrai que « si le sujet vient au travail avec ses "pré-occupations", c'est "sur-occupé" par l'activité d'autrui qu'il s'y livre... le point est décisif car on peut se demander si la fonction psychologique du travail ne réside pas précisément dans la rupture qu'il introduit entre les "pré-occupations" personnelles du sujet et les "occupations" sociales dont il doit s'acquitter » (p. 65). S'engager dans une tâche professionnelle exigerait donc cette faculté de mettre provisoirement de côté ses préoccupations personnelles pour se consacrer à celles de la tâche, bref de réordonnancer ses préoccupations et ses intentions, ce qui suppose une faculté de contrôle et de planification que l'on sait altérée chez le schizophrène (Proust, 2004).

Enfin et pour ne faire que mentionner cette dimension qui mériterait évidemment d'être beaucoup plus développée, l'insertion de toute tâche pro- 
fessionnelle dans un réseau d'activités, et par conséquent au sein d'une activité collective, conduit celui qui veut assurer cette tâche à s'insérer dans ce réseau. D'autre part, le plus souvent cette activité collective s'est stabilisée dans ses procédures et ses modes de coordination, voire s'est instituée notamment pas un mode d'organisation assignant à chacun des rôles plus ou moins définis, éventuellement aussi en stabilisant en un " genre » des pratiques et modes de coordination ; tout cela exige de l'agent l'aptitude de s'insérer (en un sens presque littéral) dans un tel dispositif très contraint, celle aussi de s'approprier l'héritage de ces « manières de faire ». Il y a des raisons de penser que ces tâches ne sont pas aisées pour un patient schizophrène. En retour, il semble clair que parvenir à s'insérer dans ce type de "poste de travail » fournit un mode privilégié et probablement sans équivalent d'intégration sociale.

Ces remarques concernent la schizophrénie, dont le cas est particulier par la connaissance assez précise des déficiences cognitives propres à cette pathologie, et qui peuvent être mises directement en rapport avec les contraintes et aptitudes requises dans les situations de travail. Le raisonnement ne peut être développé de la même manière avec la plupart des autres pathologies, dont les conséquences cognitives sont moins connues, et qui pourtant s'accompagnent aussi de difficultés d'insertion. La démarche nous semble néanmoins heuristiquement intéressante de chercher à préciser ce qui peut faire difficulté au travail, pour ces personnes présentant divers types de troubles psychopathologiques, à partir d'une analyse des situations de travail et de l'activité qu'elles impliquent. Cette voie d'investigation reste à notre sens à développer.

\section{BIBLIOGRAPHIE}

BANDuRA, A. 1994. "Self-efficacy », dans V.S. Ramachandran (sous la direction de), Encyclopedia of Human Behavior, vol. 4, New York, Academic Press, p. 71-81.

BANDurA, A. 1997. Self-efficacy : The Exercise of Control, New York, W.H. Freeman \& Co, Publishers, trad. franç. (2003) Auto-efficacité. Le sentiment d'efficacité personnelle, Bruxelles, De Boeck.

Bell, M. ; KAPLAN, E.A. ; BRYSON, G. 1997. « Cognitive impairment and work behavior in schizophrenia », Schizophrenia Research, vol. 24, Issues 1-2, p. 219.

BLANCHARD, S. 2000 « Le conseil en orientation : introduction », L'orientation scolaire et professionnelle, $29,1$.

Blanchard, S. 2003. "Le bilan de compétence ", Psychiatrie, sciences humaines, neurosciences, 1, 3, p. 31-38.

Bryson, G. ; Bell, M. ; LySAKER, P. ; ZITO, W. 1997. « The work behavior inventory : a scale for the assessment of work behavior for clients with severe mental illness », Schizophrenia Research, vol. 24, Issues 1-2, p. 220. 
CACOT, P. 2003. Quelques idées sur le positionnement conceptuel et contextuel des soins de réadaptation en psychiatrie. Communication aux États généraux de la psychiatrie, http :/ / www. eg-psychiatrie. com/sommaire. php3.

CAMPBELL, J. 1999. « Schizophrenia, the space of reasons, and thinking as a motor process », The Monist, vol. 82, 4, 609-625.

CLOT, Y. 1999. La fonction psychologique du travail, Paris, PUF.

Clot, Y. 2002. "Clinique de l'activité et répétition », Cliniques méditerranéennes, 66, 31-54.

CnAan, R.A. ; Blankertz, L.E. ; Messinger, K. ; Gardner, J.R. 1988. « Psychosocial rehabilitation : Toward a definition », Psychosocial Rehabilitation Journal, 11 (4), 61-77.

Daprati, E. ; Franck, N. ; Georgieff, N. ; Proust, J. ; Pacherie, E. ; Dalery, J. ; JeanNEROD, M. 1997. « Looking for the agent, an investigation into self-consciousness and consciousness of the action in patients with schizophrenia », Cognition, vol. 65, p. $71-86$.

Dejours, C. 2005. «Centralité du travail et santé mentale », Pratiques en santé mentale, $1,22-28$.

Evans, J.D. ; Bond, G.R. ; Meyer, P.S ; Hea Won Kim ; LysaKer, P.H. ; GibSON, P.J. ; TUNIS, S. 2004. " Cognitive and clinical predictors of success in vocational rehabilitation in schizophrenia ", Schizophrenia Research, In Press.

FLACHER, B. 2002. Travail et intégration sociale, Rosny, Bréal.

FRANCEQUIN, G. 1995. " Histoire de vie et pratique de l'orientation », L'orientation scolaire et professionnelle, 24, 301-326.

FRITH, C.D. 1992. The Cognitive Neuropsychology of Schizophrenia, Hillsdale, Lawrence Erlbaum Associates, trad. franç. La neuropsychologie cognitive de la schizophrénie, Paris, PUF, 1996.

FRITH, C.D. ; BlAKEMORE, S.-J. ; WOLPERT, D.M. 2000. « Explaining the symptoms of schizophrenia : Abnormalities in the awareness of action ", Brain Research Reviews, 31, 357-363.

GALLAGHER, S. 2000. "Self reference and schizophrenia », dans D. Zahavi (sous la direction de), Exploring the Self, Amsterdam, John Benjamins, 203-239.

GECAS, V. 1991. "The self-concept as a basis theory of motivation », dans Howard, J.A. ; Callero, P.L. (sous la direction de). The Self-Society Dynamic. Cognition, Emotion, and Action, Cambridge, NW, Cambridge University Press, 171-187.

Guichard, J. ; Huteau, M. 2001. Psychologie de l'orientation, Paris, Dunod.

Hardy-Baylé, M.-C. ; SARfatI, Y. ; PAsserieuX, C. 2003. « The cognitive basis of disorganisation in schizophrenia and its clinical correlates : Toward a pathogenetic dizorganization scale », Schizophrenia Bulletin, 29 (3), 459-471.

HonNeth, A. 2000. La lutte pour la reconnaissance (trad. franç. P. Rusch). Paris, Cerf.

JEANNEROD, M. ; FourNerET, P. 1998. «Être agent ou être agi ? Sur les critères d'autoattribution d'une action ", dans H. Grivois et J. Proust (sous la direction de), Subjectivité et conscience d'agir dans la schizophrénie et dans l'autisme, Paris, PUF.

Jolivet, B. 1995. Parcours du sanitaire au social, Éd. Frison Roche.

LALONDE, P. ; MoRIN, C. 1999. « La réadaptation dans la prise en charge de la schizophrénie », Perspectives psy, 38, 3, p. 201-206. 
LeCOMTE, T. ; LeCLeRC, C. 2004. Manuel de réadaptation psychiatrique, Québec, Presses de l'université du Québec.

LEGAY, D. 1995. "L'évaluation de l'autonomie des malades mentaux », Encéphale, 21, $425-435$.

LEGAY, D. 2001. « Les pratiques françaises de réadaptation sociale », Information psychiatrique, 77, 259-263.

LibERMAN, R.P. (sous la direction de). 1991. Réhabilitation psychiatrique des malades mentaux chroniques, Paris, Masson.

MCGuRK, S.R. ; MELTZER H.Y. 2000. « The role of cognition in vocational functioning in schizophrenia », Schizophrenia Research, vol. 45, Issue 3, p. 175-184.

MCGURK, S.R. ; MUESER, K.T. 2004. « Cognitive functioning, symptoms, and work in supported employment : a review and heuristic model », Schizophrenia Research, in Press.

MEARA, N. ; PAtTon, M. 1994. " Contribution of the working alliance in the practice of career counseling », The Career Development Quartely, 43, p. 161-177.

PROUST, J. 2000. "Awareness of agency : Three levels of analysis », dans T. Metzinger (sous la direction de), The Neural Correlates of Consciousness, Cambridge, MIT Press, 307-324.

Proust, J. 2003. " Thinking of oneself as the same », Consciousness and Cognition, 12, 4, 495-509.

PROUST, J. 2004. "Agency in schizophrenia from a control theory viewpoint ", dans W. Prinz \& N. Sebanz (sous la direction de) Disorders of Volition, Cambridge, MIT Press (in press).

REVICKI, D.A. 1998. "Quality of life and re-integration of chronically ill patients », European Psychiatry, vol. 13, Supplement 4, p. 219.

SARfati, Y. ; HaRdy-BAyle, M. ; BRUnet, E. ; WildlöCher, D. 1999. " Investigating theory of mind in schizophrenia : Influence of verbalization in disorganized and non-disorganized patients », Schizophrenia Research, 37(2), 183-190.

TESSIER, L. ; CLÉMENT, M. 1992. La réadaptation sociale en psychiatrie : défi des années 1990, Québec, Gaétan Morin Ed.

TORRENTE, J. 2004. Le psychiatre et le travailleur. Cheminement de la psychopathologie du travail d'hier à demain, Paris, Doin.

VIDON, G. (sous la direction de). 1995. La réhabilitation psychosociale en psychiatrie, Paris, Éditions Frison-Roche.

VIDON, G. ; DubuIS, J. ; LEGUAY, D. (sous la direction de). 1999. « La réhabilitation psychosociale en France : état des lieux », L'information psychiatrique, 75, 4, p. 347-416.

WildER-WILlis, K.E. ; SHEAR, P.K. ; STEFFEN, J.J. ; BORKIN, J. 2002. « The relationship between cognitive dysfunction and coping abilities in schizophrenia », Schizophrenia Research, vol. 55, Issue 3, p. 259-267.

\section{Résumé}

La réinsertion professionnelle des personnes ayant présenté des troubles psychopathologiques fait actuellement l'objet d'une diversité de pratiques d'accompagne- 
ment et de soutien, essentiellement développées dans le secteur médico-social. Les problèmes d'articulation entre le monde des soins, le secteur médico-social et le monde du travail, nous conduisent à rapporter l'hétérogénéité de ces mondes au type d'activité qui les fonde et à argumenter que la tâche de soutien à la réinsertion étant radicalement distincte de celle des soins, il paraît préférable de la développer et de la théoriser dans un cadre propre. Nous caractérisons ensuite notre expérience des bilans d'aptitude au travail et d'orientation, et tentons de préciser quelques dimensions pertinentes pour étayer cette appréciation du potentiel d'insertion. Nous indiquons enfin quelques directions de recherche et arrière-plans théoriques pour développer une réflexion théorique spécifique à cette problématique de l'insertion au travail des personnes psychopathologiquement vulnérables.

Mots-clés

Réinsertion professionnelle, handicap psychique, bilan d'évaluation, orientation professionnelle, fonction psychologique du travail.

PSYCHOPATHOLOGY AND VOCATIONAL REINTEGRATION :

A MULTIDIMENSIONAL APPROACH REQUIRING MULTIDISCIPLINARY THEORETICAL RESOURCES

\section{Summary}

The vocational reintegration of people suffering from psychopathological disorders is presently supported by a variety of services mainly developed in the medico-social field. The problems of coordination between the medical world, the medico-social field and the vocational world lead us to see the heterogeneity between these worlds as a result of their deeply different basic activity. Consequently, we argue that, given the difference between care and the task of supporting reintegration, it seems preferable to develop the later and to theorize about it in its own field. Then, we characterize our experience of assessments of work ability and for vocational counselling. We try to specify few relevant dimensions with regards to the reintegration potential. Finally, we sketch few lines of research and theoretical backgrounds in order to further develop a specific theoretical thinking about vocational reintegration of people with a psychopathological vulnerability.

Keywords

Vocational reintegration, psychic disability, assessments of work ability, vocational counselling, psychological function of work. 